This is a post-peer-review, pre-copyedit version of an article published in Microfluidics and Nanufluidics.

The final authenticated version is available online at: https://doi.org/10.1007/s10404-017-1875-x

\title{
A simple droplet merger design for controlled reaction volumes
}

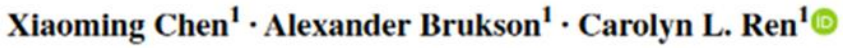

Abstract Droplet microfluidics has been proven to be a viable platform for a variety of reactions by using uniform droplets well encapsulated by an immiscible carrier medium as reaction vesicles. Merging as one of the most basic forms of droplet manipulation is highly demanded in controlling the concentration of reagents for reactions. After preliminary testing of several reported passive merging designs, it is found that most of them are prone to fabrication and operation uncertainties which limit their appli-

dimensions according to the desired set of reactions. The simplicity of the design allows for easier design and configuration to a desired purpose and lows down the risk of failures due to fabrication and operation uncertainties. The single-input-single-output design minimizes pressure disturbances from propagating downstream and reduces the complexity of integration into a larger LOC system. The geometry can be scaled up with little to no observable effect on the mode of operation.
\end{abstract} cation for practical use. In this work, we present a simple design for merging variable numbers of droplets with a valve-type function which enables a critical volume limit to be imposed to ihe final product droplet. When the product droplet reaches the critical volume limit, it will block the exit of the merging chamber serving as the close mode of the valve. As a result, the pressure upstream is built up which forces the product droplet to exit the merger. The relationship between the numbers of droplets merged, the length of input droplets and the length of output droplets is used to tune the design parameters such as the chamber length, pillar array dimensions and bypass channel
Keywords Microfluidics · Droplets · Droplet merger

\section{Introduction}

Over the past decade, the field of droplet microfluidics has advanced significantly (Tabeling 2014). The interest in droplets as a viable platform for processing chemical reactions has grown with each year. The use of droplets has found applications in a variety of fields from nanoparticle synthesis (Hung et al. 2006; Frenz et al. 2008), drug screening (Miller et al. 2012), tissue engineering (Xiong et al. 2014), biomedical diagnostics (Kaler and Prakash 2014), single-cell encapsulation (Basu 2013; He et al. 2005), DNA amplification (Wheeler et al. 2003) and directed evolution (Guo et al. 2012), which speaks to the immense interest in this technology (Lorenz et al. 2008).

Lab-on-a-chip (LOC) devices can incorporate several different tools which have been developed for manipulating droplets such as droplet generation (Garstecki et al. 2006; van Steijn et al. 2010; Glawdel et al. 2012), sorting (Glawdel et al. 2011), splitting (Link et al. 2004), trapping (Dewan et al. 2012), sensing (Isgor et al. 2015), heating (Boybay et al. 2013) and storage of droplets (Trivedi et al. 2010) on or off chips. One of the critical components 
for droplet-based LOC devices to be a viable platform for high-throughput screening is to reliably combine and mix the desired reagents. Some methods for introducing reagents into droplets include co-flowing, where reagents are flowed in parallel prior to droplet generation (Song et al. 2003); direct injection (Abate et al. 2010), where a small amount of fluid from a perpendicular channel is allowed to merge with a passing droplet; and droplet merging (Bremond et al. 2008; Niu et al. 2008; Christopher et al. 2009; Yang et al. 2011; Mazutis et al. 2009), where two or more droplets are allowed to fuse together. Merging droplets allows for a controlled method of combining reagents in a way that allows the contents to mix quickly without the risk of cross-contamination.

In general, the different methods for merging droplets are divided into active and passive methods. Active methods are those which employ an external force such as electrical (Lee et al. 2014; Xu et al. 2012), magnetic (Gu et al. 2011), thermal (Luong et al. 2012), pneumatic controls (Yoon et al. 2014; Lin and Su 2008) or optical (Li et al. 2011), while passive methods (Bremond et al. 2008) use the channel geometry to manipulate the flow in a particular manner. Fach kind of method has pros and cons. Active designs usually allow for on-demand controls, but require external components such as power sources, valves or control units which make the operation of the device increasingly complex and increase the overall cost of the system. Although passive designs have not been largely implemented in practical applications due to their sensitivity to fabrication and operation uncertainties, their simplicity of operation, minimal external equipment, no active control, coupled with potentially high throughput could be an excellent alternative for reliable droplet-based microfluidic devices. Therefore, the following will focus on passive merging structures.

Some passive designs incorporate a channel expansion (Xiong et al. 2014; Tan et al. 2004) to remove the oil separating adjacent droplets and thus reduce their distance so that the adjacent droplets can collide and merge. Similarly, other implementations have used cross-junctions to partially remove the oil phase separating adjacent droplets in microchannels (Tan et al. 2007). These methods have been shown to be effective in merging droplets, but require ensuring the droplets are within an appropriate spacing and the designs which remove (instead of redirecting) the oil phase can cause pressure fluctuations downstream (Niu et al. 2008), which make the system prone to fabrication and operation uncertainties. Other methods include forcing droplets to merge through the use of velocity gradients (Hung et al. 2006; Liu et al. 2007; Jin et al. 2010). A long channel with increasing width creates a velocity gradient along the channel, and different numbers of successive droplets have been shown to merge (Hung et al. 2006; Liu et al. 2007). The difference in velocity between droplets of different sizes and viscosities has also been utilized to collide and merge droplets in microchannels (Jin et al. 2010). The use of velocity gradients can be more effective than a straight channel expansion, but however, poses the risk of unwanted droplet coalescence or droplets not remaining in contact long enough to actually merge. Another common technique for merging droplets utilizes channel constrictions to generate a Laplace pressure difference across the downstream and upstream ends of the droplet. In combination with a bypass channel filled with the continuous phase (i.e., oil), the droplets are slowed or trapped, allowing other droplets to collide and merge (Niu et al. 2008; Venkatachalam et al. 2010). In particular, Niu et al. (2008) were able to successfully trap and merge droplets in a droplet train by using pillar structures to facilitate droplet trapping and oil bypass. This geometry allows droplets to be trapped for an extended period of time and does not have any observable effects on the inter-droplet spacing. Although the design has been shown to be highly effective in merging droplets, it was not shown the conditions under which the chamber can merge different numbers of droplets under controlled manner. We attempted this design at the beginning with limited success in robust performance due to the difficulty in accurately predicting the required pressure drop for releasing the merged droplet. The pressure drop is influenced by the three-dimensional droplet shape which is unknown based on two-dimensional imaging, and it is sensitive to any defects in channel dimensions because it is inversely proportional to the cube of the channel width. Oil often swells PDMS which always causes deviations in the designed channel dimensions and cause deviation in the predicted pressure drop. In addition, it is found that the large exit in their design also poses challenges to merging because the merged droplet cannot block the exit to increase the resistance of the bypass channel. As a result, the following droplets would flow through the bypass channels causing undesired merging events.

In this work, we propose a passive merging chamber capable of merging variable numbers of droplets by designing a valve-type function in the merging chamber. The relationship between the numbers of droplets merged, the length of input droplets and the length of output droplets is used to tune the design parameters according to the desired set of reactions. The merging is merely dependent on the channel geometry which is much more robust. The reduced complexity of the design allows for easier design and configuration to a desired purpose and low risk of failures due to fabrication and operation uncertainties. The single-input-single-output design minimizes pressure disturbances from propagating downstream (Niu et al. 2008) and reduces the complexity of integration into a larger LOC system. The specific geometry of the chamber allows for 
the operation of a valve-type function to force the droplet from the chamber, thereby imposing a critical volume limit. The geometry can be scaled up with little to no observable effect on the mode of operation.

\section{Design of the merging chamber and its working principle}

\subsection{Design of the merging structure}

Figure 1 is the schematic of the proposed merging design. There are two columns of pillars that act to divide the merging chamber into three virtual channels - two bypass channels and one central channel where droplets are designed to merge. The dimensions labeled are: $L$, the chamber length; $W_{\text {by }}$, the width of the bypass channel; $W_{\mathrm{c}}$, the width of the central chamber between the pillar arrays; $W_{\mathrm{s}}$, the distance between each pillar; $W_{p}$, the width of each pillar; and $L_{p}$,

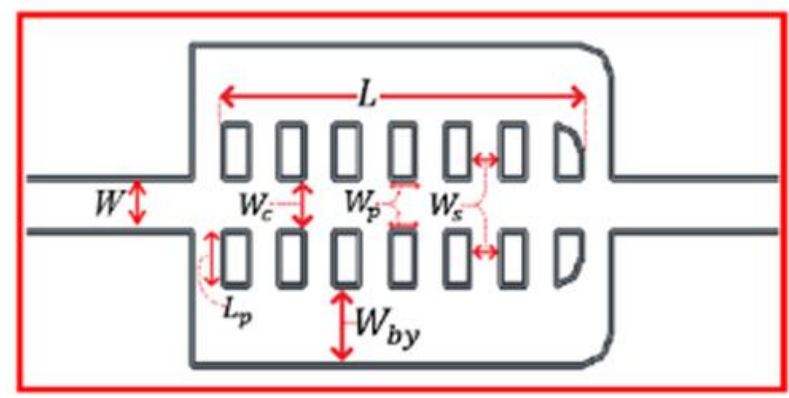

Fig. 1 Schematic of the merging chamber. The labeled dimensions are $I_{\mathrm{s}}, W_{\mathrm{by}}, W_{\mathrm{c}}, W_{\mathrm{p}}, W_{\mathrm{s}}$ and $I_{\mathrm{p}}$, which represent the chamber length, bypass channel width, width of the central chamber, width of the pillars, inter-pillar spacing and pillar lengths the length of each pillar. Each dimension is normalized with the upstream channel width $W$, which is the same distance between the two pillar arrays.

$$
L^{+}=\frac{L}{W}, W_{\mathrm{by}}^{+}=\frac{W_{\mathrm{by}}}{W}, W_{\mathrm{p}}^{+}=\frac{W_{\mathrm{p}}}{W}, W_{\mathrm{c}}^{+}=\frac{W_{\mathrm{c}}}{W}, W_{\mathrm{s}}^{+}=\frac{W_{\mathrm{s}}}{W}, L_{\mathrm{p}}^{+}=\frac{L_{\mathrm{p}}}{W}
$$

The design of the chamber is such that the entering droplets are allowed to be trapped in the central channel (instead of the bypass channel) and the oil is forced to bypass the droplet via the bypass channels. Once the merged droplet has reached the volume limit threshold, it will extend through the pillar array and block the exit of the bypass channel, preventing the oil from bypassing the droplet. As a result, the pressure upstream will be built up which will push the merged droplet to exit the chamber.It is important to design the chamber structure such as the central channel width, pillar spacing, chamber length and bypass channel dimensions based on the needs, for instance, the input droplet width and the desired number of droplets to be merged. When the droplet width is less than the channel width which is also the same as the central path of the merging chamber (i.e., droplet is not confined by the channel walls), it tends to pass directly through the merging chamber without being affected and thus trapped. In order to be merged, droplets need to have a width larger than the designed channel width. When such a "large" droplet enters into the merging chamber, it will expand and consequently occupy some of the space between the pillars (Gu et al. 2011), because the surface tension tends to minimize its surface area making it as circular as possible. This is also observed in Fig. 2 where the droplets within the pillar array have a small but noticeable extension into the inter-pillar spacing. The presence of the droplet coupled with the droplet curvature between the pillars increases the resistance along

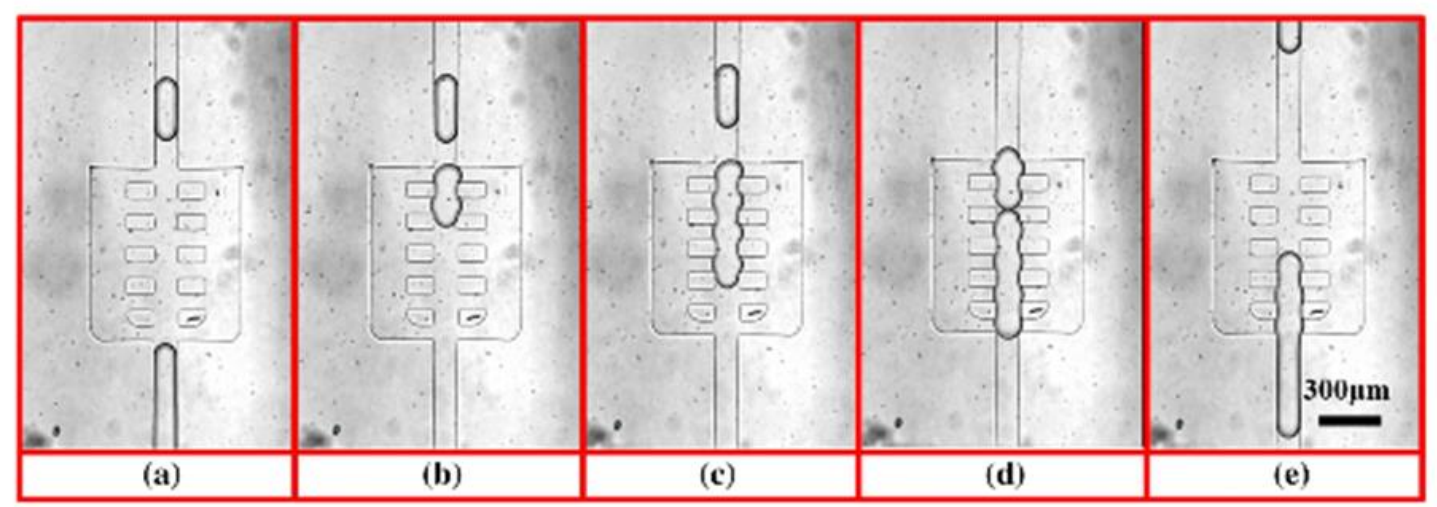

Fig. 2 Sequential droplet merging. a The droplet enters the merging chamber, $\mathbf{b}$ the first droplet is trapped between the pillars allowing the next droplet to catch up, $\mathbf{c}$ the first two droplets merge and remain trapped within the pillar array and the length of the product droplet increases proportionally, $\mathbf{d}$ the product droplet blocks the exit of the bypass channel when the final droplet enters the central chamber, $\mathbf{e}$ the product droplet is fully merged and is forced out of the chamber. Scale bar is $300 \mu \mathrm{m}$ 
the central path and forces oil to bypass the droplet. This mechanism acts to slow the droplet and trap it between the pillars allowing the oil to bypass the droplet and the following droplets to catch up and merge together. The product droplet will remain trapped between the pillars until its volume reaches a critical threshold; the critical threshold is determined by the chamber length, $L^{*}$. At this critical threshold, the product droplet will block the exit of the bypass channel and generate a large pressure buildup near the entrance of the chamber causing the droplet to be forced out of the chamber.

Robustness has been one of the key issues with mergers which require optimization for each design parameter. For example, once within the chamber, the curving of the droplet between the pillars will slightly reduce the length of the droplet which could alter the expected results for the merging device reducing its robustness. Therefore, the spacing between the pillars must be optimized to minimize the deformation of the droplet while maximizing the capacity of the oil to bypass the droplet. The spacing between the pillars $\left(W_{\mathrm{s}}^{*}\right)$ is set to a constant value of 0.625 in this study. It was determined as the robust spacing in order to adequately prevent droplets from entering the chamber's bypass channel while still allowing sufficient oil to bypass through and providing the appropriate amount of space for the droplet to expand between the pillars. The length of the pillars $\left(L_{p}^{*}\right)$ is set to 1 to have the pillar length greater than 1.5 times $W_{p}^{*}$; this is to ensure that the pillar structures do not become deformed or incomplete during the fabrication procedure. The width of the central channel $\left(W_{\mathrm{c}}^{*}\right)$ is set to unity in order to minimize the alteration of the droplet length within the pillar array. Finally, the pillar width $\left(W_{p}^{*}\right)$ is set to 0.5 which was determined as the minimal pillar width to ensure the quality of the microstructures after the fabrication process. If the width/height ratio of pillars is too small, the pillars might be peeled off during the fabrication process. The remaining, $W_{\text {by }}^{*}$ and $L^{*}$ are then calculated to the desired criteria of the LOC device.

Figure 2 shows the procession of droplet merging from the time before the droplet enters the chamber, to the first droplet becoming trapped between the pillars, the sequential merging of droplets in the train, the blocking of the bypass channel exit and finally, the product droplet releases after reaching the critical volume threshold. A video attached in the supplemental material shows the detailed droplet merging process. This behavior is advantageous as it provides a passive on/off mechanism of operation which can be useful for predictable operation under different conditions. This function is most useful in preventing the undesired merging of reagents by ensuring the product droplet is released in synchrony with the following droplet entering the channel.

\subsection{Impact of the design parameters on merging performance}

The extension of the product droplet length within the central pillar array is proportional to the individual droplets merged and is limited by the imposed critical volume limit. Therefore, the length of the droplets entering the chamber will have a direct impact on the operation of the merging chamber. The geometry of the merging chamber will influence two key parameters: the critical volume limit, and the critical capillary number which shows the competition between the viscous force and interfacial tension force. Changing the length of the merging chamber will proportionally change the critical volume threshold by altering the length which the product droplet can extend. And at the same time, changing the length of the chamber in combination with the bypass channel width will influence the capability of the oil to bypass the droplet through altering relative channel resistances. Then, due to the balance of the surface tension and viscous forces, there exists a regime where the surface tension force dominates in holding the droplet within the pillar array and allowing the product droplet to reach the critical volume threshold. Conversely, there also exists a regime where the viscous forces of the oil overcome the capability of the droplet to become trapped and the merging cannot be predicted. The transition between these two regimes is the critical capillary number. Therefore, the effects of the droplet length, geometry, inter-droplet spacing and capillary number are studied to characterize the modes of operation for the proposed merging design.

\subsubsection{Effect of droplet length}

From the geometry, the critical volume threshold will occur at a volume between the $N^{\text {th }}$ droplet and the $(N-1)^{\text {th }}$ droplet merged within the pillar array. Benefiting from the confinement to the droplet height and width by the channel dimensions, any increase in the droplet volume will be reflected by an increase in the droplet length. Therefore, it can be determined that the length of the droplet exiting the channel $\left(I_{0}\right)$ should be an $N$ multiplier of the input droplet length $\left(L_{\mathrm{d}}\right)$, which will be larger than or equal to the length of the chamber $(L)$. Referring to Fig. 1, the chamber length is defined as the spacing between the first and last pillar and the trapped droplet should have a length less than the chamber. This gives us the inequality equation:

$(N-1) L_{d}<L<N L_{d}$

Rearranging Eq. (1), we can obtain the number of droplets that can be trapped in a chamber with a length of $L$, 
$\frac{L}{L_{d}}<N<\frac{L}{L_{d}}+1$

By substituting $N=\frac{L_{0}}{I_{d}}$ into Eq. (2), the range of the output droplet length $\left(L_{0}\right)$ can be derived.

$1<\frac{L_{0}}{L}<\frac{L_{d}}{L}+1$

Using Eqs. (2) and (3), we can predict the number of droplets which can be merged based on the priority of the desired variable: With a desired volume of droplet (fixed $L_{\mathrm{d}}$ ), Eq. (2) can be used to predict the number of droplets which are possible to be merged and adjust chamber length, $L$, in order to achieve the desired $N$. Conversely, with a predetermined number of droplets to be merged (fixed $N$ ), the range of $L_{d}$ can be determined to best optimize the flow conditions for a fixed $L$.

\subsubsection{Effect of geometry}

The effect of the parameters $L^{*}$ and $W_{\mathrm{by}}^{*}$ (non-dimensional form) was analyzed by using a simplified flow resistance model where the pillars act to divide the merging chamber into three channels (i.e., two bypass channels and one central channel). This is illustrated in Fig. 3 where the flow resistance model is comprised of the parallel addition of resistors. The ratio between the total bypass flow resistance $R_{\text {by }}$ and the flow resistance of the central channel $R_{\mathrm{c}}$ is proportional to the ratio of oil flowing through each branch and can be estimated using the channel dimensions. The bypass resistance ratio (BRR) is represented as:

$\mathrm{BRR}=\frac{R_{\mathrm{by}}}{R_{\mathrm{c}}}=\frac{R\left(h, W_{\mathrm{s}}, L_{\mathrm{P}}\right)+\frac{1}{2} R\left(h, W_{\mathrm{by}}, L\right)}{R(h, W, L)}$

where $R$ is the function which estimates the hydrodynamic resistance of laminar flow in a straight rectangular channel with a height of $h$, width of $w$ and length of $L$, with a constant fluid viscosity of $\mu$, which can be expressed as (White 1991):

$R_{\mathrm{h}}=\frac{12 \mu L}{w h^{3}}\left[1-\frac{h}{w}\left(\frac{192}{\pi^{5}} \sum_{n=1,3,5}^{\infty} \frac{1}{n^{5}} \tanh \left(\frac{n \pi w}{2 h}\right)\right)\right]^{-1}$

The bypass resistance will change depending on the amount of side channels which are available for oil to pass through; the BRR is calculated using the maximum $R_{\text {by }}$ possible (i.e., when the droplet occupies the maximum amount of space before the critical volume threshold) in order to make sure droplets will enter the central channel when droplets arrive at the junctions. This is predicted to occur when the first pair and the last pair of side

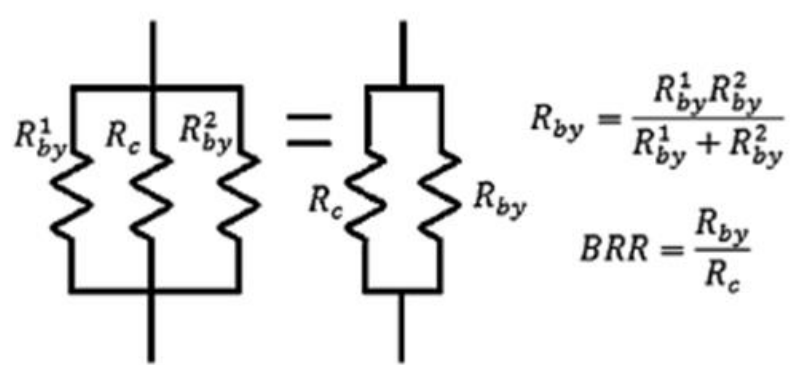

Fig. 3 Equivalent flow resistance model for the chamber. The pillar arrays act to separate three parallel fluid channels which can be simplified as two channels for oil bypass and droplet merging

channels are accessible and the remaining side channels are blocked. The BRR represents the ratio of the flow rates through the bypass and the central channels. The advantage of this approach (as opposed to the ratio of the total flow rate) is that it conveniently sets a ceiling such that the resistance of the central channel is always larger than that of the bypass channel $(B R R<1)$.

\subsubsection{Effect of capillary number}

The functionality of the merging chamber is dependent upon the competition between viscous and surface tension forces. The ratio of these two forces is represented by the capillary number $(\mathrm{Ca}=\mu U / \gamma$, where $\mu$ is the fluid viscosity, $U$ the average velocity of the continuous phase, and $\gamma$ the interfacial tension). Surface tension force acts to prevent the droplet from easily passing through the pillar array and even trapping the droplet, while viscous force tends to push the droplet through the pillar array without stopping. Therefore, there exists a critical capillary number of the continuous phase liquid $\left(\mathrm{Ca}_{\text {crit }}\right)$ at which the droplets can no longer be trapped because the pressure exerted on the droplet from the oil flow will exceed the maximum possible drag force the droplets experience in the pillar array.

The efficacy of the design is tested by varying the length of input droplets and comparing to the predicted results from Eqs. (2) and (3). The designs were chosen such that for a fixed droplet length $\left(L_{\mathrm{d}} / W \approx 2.2\right)$, the chamber would merge two, three, four and six droplets with chamber length, $L^{*}$, equal to $3.2,5.5,8.0$ and 13.0, respectively. Also, the relationship between the BRR and $\mathrm{Ca}_{\text {crit }}$ is examined by varying the bypass channel width, $W_{\text {by }}^{*}$, which is set to be 1.5 and 3.1 , respectively, for each chamber length, $L^{*}$ (i.e., eight designs in total). An empirical model to determine the working flow regimes is developed based on experimental data. 


\section{Experimental validation}

\subsection{Materials and methods}

The microfluidic chips were fabricated from polydimethylsiloxane (PDMS) using standard soft lithography methods. The height $(h)$ of the microchannel was set to be $60 \mu \mathrm{m}$ with a width $(W)$ of $120 \mu \mathrm{m}$. The fabrication of the master and subsequent PDMS molding is described elsewhere (Liu et al. 2008). Briefly, negative photoresist (SU-8, Micro Chem) is spin-coated on the surface of a clean silicon wafer and patterned using high-intensity UV light and a photomask. Then, PDMS is poured over the wafer and baked at $95{ }^{\circ} \mathrm{C}$ for at least $3 \mathrm{~h}$. The resulting PDMS slab is cut and bonded to a PDMS-coated glass slide using oxygen plasma bonding.

The continuous phase used in all experiments was silicone oil (10 cSt, Sigma-Aldrich) with no surfactant, and the dispersed phase used was pure water. The interfacial tension between silicone oil and pure water is $\gamma=42 \mathrm{mN} / \mathrm{m}$. Since silicone oil swells PDMS, the actual channel height was measured as $53 \mu \mathrm{m}$, and channel width $110 \mu \mathrm{m}$ after sufficient swelling. The fluids were driven using syringe pumps (Pump 33, Harvard Apparatus) at oil flow rates between 60 and $220 \mu \mathrm{l} / \mathrm{hr}$ and flow rate ratio $\left(Q_{\mathrm{w}} / Q_{\mathrm{o}}\right.$, where $Q_{\mathrm{w}}$ is the flow rate of water and $Q_{O}$ is the flow rate of silicone oil) between 0.1 and 1.5 for one set of experiments. A pressure system was used in combination with flow sensors for the second set of experiments. The measured flow rates from the flow sensor were between 60 and $360 \mu \mathrm{l} / \mathrm{hr}$. Although syringe pumps have been shown to have more variability in the flow rate compared to pressure systems, syringe pumps were chosen because the system was designed to have a large hydrodynamic resistance and therefore would require pressures outside the range of our current pressure system which is 1 bar. The pressure system was later introduced to alleviate the long flow transients experienced by syringe pumps when changing flow rates. Droplets were imaged using the Nikon Eclipse $\mathrm{Ti}$ inverted microscope with a QImaging (Q24814) camera with varying capture rates between 150 and 500 frames per second. The droplet motion was captured at a variety of frame rates, and the videos were analyzed for a variety of droplet parameters using droplet morphometry and velocimetry (DMV) software (Basu 2013).

\subsection{Experimental procedure}

The syringes were connected to the chip by PEG tubing inserted into reservoir holes in the PDMS slab. The syringe pump was operated manually with oil flow rate between 60 and $220 \mu \mathrm{L} / \mathrm{hr}$. The water flow rate was varied as a ratio to the oil flow rate between 0.1 and 1.5. Silicone oil was pumped into the microfluidic chip for $40 \mathrm{~min}$ to sufficiently swell the PDMS channel before generating droplets, following the measurement of channel dimensions. After that, droplets were generated in a $\mathrm{T}$-junction geometry with a single oil channel and a single dispersed phase channel with a width ratio of 1 to ensure the droplet generation process works in the squeczing regime in a larger flow rate range (Garstecki et al. 2006). The droplet lengths generated were between $144 \pm 12$ and $300 \pm 11 \mu \mathrm{m}$ (i.c., $0.17<L_{\mathrm{d}} / L<0.66$ ). The merging of droplets was observed for prolonged periods of time (up to $30 \mathrm{~min}$ ) to confirm the stability of the flow as well as of the design. Additionally, an oil dilution stream was used to observe the effect of input droplet spacing on the design. The results were plotted in comparison with the predictions previously described.

Finally, $\mathrm{Ca}_{\text {crit }}$ was determined by incrementally increasing the continuous phase flow rate phase until the droplets were no longer capable of being trapped within the pillar array. In order to accurately determine the range for the critical capillary number, a pressure system and flow sensors were used in order to alleviate long transient effects prevalent in syringe pumps; the flow sensor is capable of determining flow rates for silicone oil between 30 and $360 \mu \mathrm{l} / \mathrm{hr}$ with an accuracy of $\pm 3 \mu \mathrm{L} / \mathrm{hr}$. The critical capillary number was determined to be between $9.1 \times 10^{-4}$ and $2.5 \times 10^{-3}$ for the range of merging designs tested.

\subsection{Results and discussion}

To test the operating conditions of the chamber, droplet trains of different lengths were generated on chip. By generating droplet trains of variable droplet lengths, it was observed that the number of droplets which can be merged in a single chamber can be tuned for a desired purpose. Larger droplet lengths result in less droplets merging within the chamber, as expected from the theoretical model. Alternatively, droplet trains were generated at a fixed droplet length with incrementally increasing oil flow rates to determine the transition from droplet trapping to non-trapping, characterized by $\mathrm{Ca}_{\text {crit }}$.

\subsubsection{Effect of droplet length}

The number of droplets which can be merged in a single chamber can be predicted based on the length of the input droplets using Eq. (2). With varying water-to-oil flow rate ratio $(\varphi)$ and thus varying the input droplet length, it was observed that different numbers of droplets can be merged by adjusting their lengths relative to the chamber length $\left(I_{d} / L\right)$. Figure 4 a shows the number of droplets merged as a 
function of the input droplet length-to-chamber length ratio, while Fig. $4 \mathrm{~b}$ shows the output droplet length as a function of the input droplet length, both as a ratio to the merging chamber length. The thin black dashed lines represent the theoretical limits based on Fqs. (2) and (3), respectively. The hyperbolic shape of the limits for $\mathrm{N}$ in Fig. 4a provides a design guidance of the chamber length for a required $\mathrm{N}$. When a large $\mathrm{N}$ is required, it also requires for the range of $L_{\mathrm{d}} / L$ to decrease, and conversely, as $\mathrm{N}$ decreases the range for $L_{d} / L$ increases. For simple reactions involving 2-3 reagents, the operating systems (pressure systems, syringe pumps, etc.) need not be controlled precisely because of the
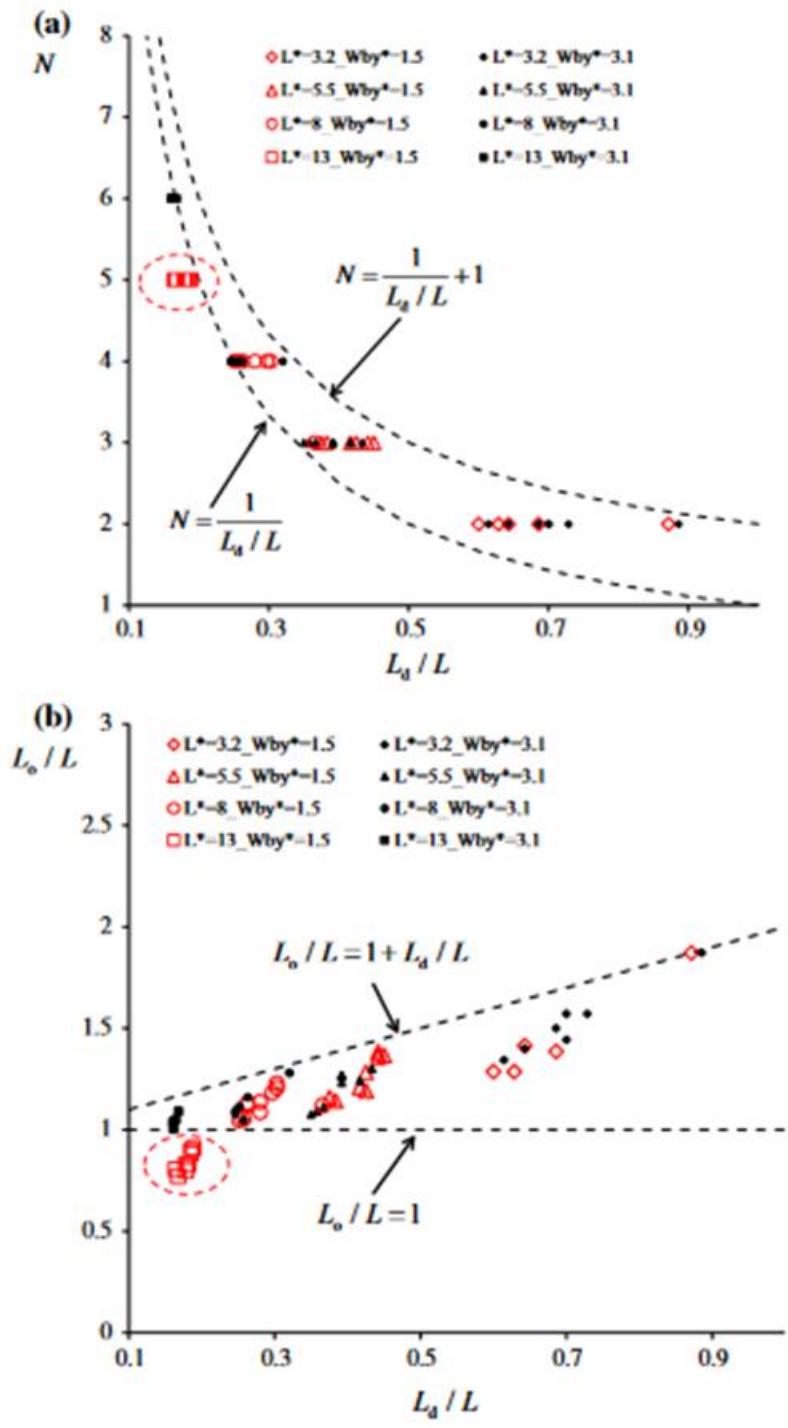

Fig. 4 a The observed number of droplets merged as a function of $L_{\mathrm{d}} / L$; b the output droplet length as a function of $L_{\mathrm{d}} / L$ . The black dashed lines represent the upper and lower limits from Eqs. (2) and (3), respectively. Eight geometries were tested with $L^{*}=3.2,5.5,8.0$, and 13 , and $W_{b y}^{*}=1.5$ and 3.1 larger acceptable range for $\mathrm{L}_{\mathrm{d}} / L$. The design compensates for increasingly complex reactions by reducing the range of $L_{\mathrm{d}} / L$; typical microfluidic systems are capable of producing highly monodispersed droplets under a variety of conditions. At the same time, increasing the number of droplets to be merged also narrows the predicted range for $L_{0}$ as shown in Fig. 4b. When it is necessary to work with precise fluid volumes, the narrow range for $L_{0}$ can allow for precise calculation of concentrations and can climinate deviations due to reaction volume dependence. The results of Fig. $4 \mathrm{~b}$ show that the output droplet length can be controlled within a narrow band regardless of the number of droplets merged at low droplet lengths. This would allow for the reaction concentration to be accurately controlled by controlling the total reaction volume.

From Fig. 4a and b, one can see that most of the experimental data fall into the range of the upper limit and lower limit except one geometry with chamber length $L^{*}=13$, bypass channel width $W_{t y}^{*}=1.5$ (hollow square) as marked in the dashed red circle. This merging design has a long chamber and narrow bypass channel, and thus a very high resistance in the bypass channel (i.e., BRR is large in this geometry). The product droplet will be pushed out of the central channel before it merges enough droplets to block the bypass channel exit. When the bypass channel width is increased to be $W_{b y}^{*}=3.1$ (solid square in Fig. 4), BRR is largely decreased and the geometry can merge six droplets as predicted. Therefore, the chamber length determines the number of merged droplets $\mathrm{N}$, and BRR determines the working robustness of this merging design. The following section will discuss the influence of BRR on critical capillary number.

\subsubsection{Effect of capillary number and geometry}

The operation of the merging design is dependent upon the competition between the viscous force and surface tension force; the ratio of these two forces is represented by the capillary number. The oil phase flow rate was varied from 60 to $360 \mu \mathrm{l} / \mathrm{hr}$ to expand the range of capillary number while maintaining the remainder of the parameters. The droplet merging process was captured by a high-speed camera.

As the BRR increases, the proportion of oil which will tend to flow through the central channel increases. Therefore, it is expected that $\mathrm{Ca}_{\text {crit }}$ will decrease for increasing BRR. Figure 5 shows where the transition occurs from trapping to non-trapping for the different designs tested, indicated by the blue (trapping) and red (nontrapping) markers. It is visible that as the BRR increases, the critical capillary number decreases; as expected, the decreased proportion of oil which is able to flow through the bypass channel results in the pressure exerted on the 
Fig. 5 Map of the operational regimes for droplet trapping. The oil flow rate was incrementally increased until the slipping of the droplet within the pillar array was observed. It was determined that the critical capillary number occurs at the capillary number between the observed trapping points (blue) and the observed slipping point (red). For the determination of the effect of the capillary number, channel lengths of $L^{*}=3.2$, 5.5 and 8 were used with $W_{b y}^{*}=$ 1.5 and 3.1 (color figure online)

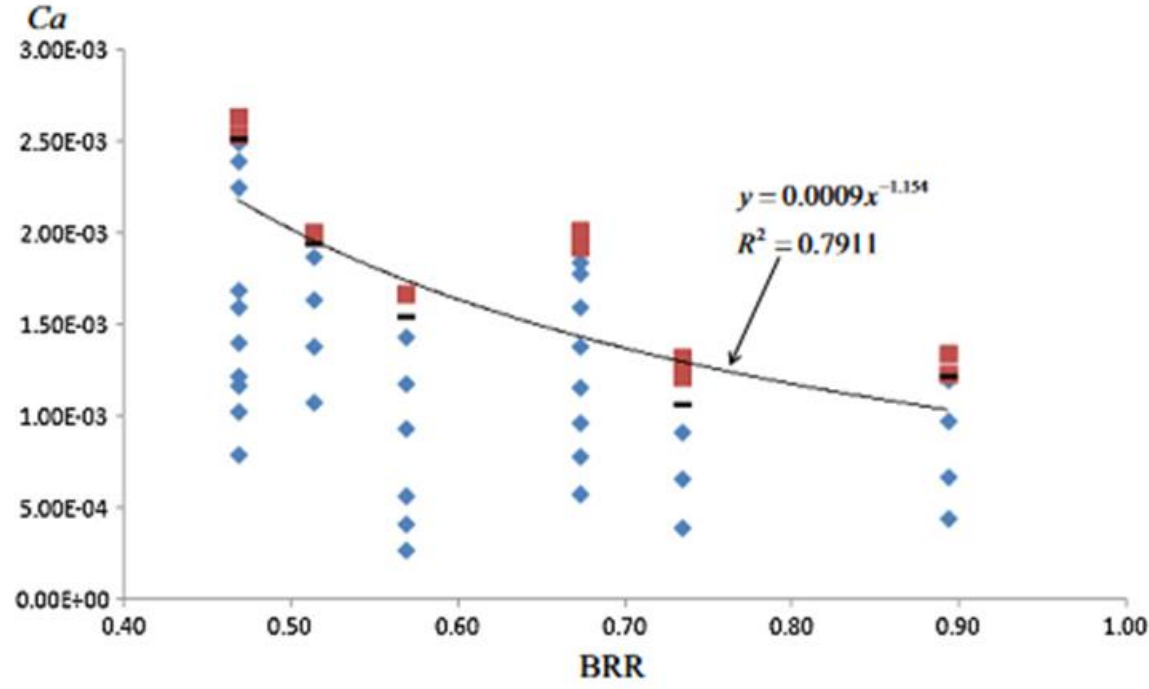

droplets to increase, preventing the droplets from merging. In this case, the viscous forces of the oil phase overcome the surface tension forees which act to hold the droplet in place. The droplet therefore is slowed down through the pillar array but not trapped; when the droplet is slowed as such, the inter-droplet spacing can be adjusted to potentially still achieve the desired droplet merging until the relative velocity of the droplet within the pillar array cannot be overcome by the drag forces.

A single outlying point in Fig. 5 does not follow the expected trend for the relationship between the capillary number and the BRR. The potential cause of this outlier is due to unfavorable wetting of the dispersed phase onto the channel walls. When this unfavorable wetting occurs, the droplet will have an increased affinity to interact with the PDMS walls. This interaction is observed as a "sticking" of the droplet to the PDMS walls and contributes to the force balance occurring in the pillar array. It causes the droplets to be able to resist a higher capillary number than otherwise would be expected, therefore creating the outlying data point.

For a specific merging design in an application, the required number of merged droplet $\mathrm{N}$ and droplet size are usually fixed. Therefore, the chamber length $\mathrm{L}$ is also fixed. In this case, one can enlarge the bypass channel width $W_{\text {by }}$ to make the design work in a larger capillary number range. On the other hand, the bypass channel width cannot be enlarged too much. Otherwise, some aqueous liquid may go into the bypass channel and stay at the corner forever (dead volume), which will affect the performance of the merging design.

\subsubsection{Effect of spacing}

The effect of droplet spacing increase was tested by adding a diluting stream to increase the volume of oil between each pair of droplets. Droplets were generated at a constant flow rate ratio $\varphi$ in order to generate droplets of comparable sizes between each trial. When the droplet spacing is large (>3 times droplet length), the droplets are capable of being trapped but at higher capillary numbers (larger than critical capillary number), do not remain trapped long enough for the next droplet to reach the chamber for them to merge. When the spacing of the droplets was reduced (1-3 times droplet length), it was observed that the functionality of the merging chamber was not affected and the merging adhered to the inequalities previously discussed. Reducing the spacing ( $<1$ times droplet length) resulted in undesired merging for low droplet lengths $\left(L_{\mathrm{d}} / L<0.3\right)$, but there was no observable effect for larger droplets. The droplet spacing does not have a considerable effect on the operation of the merging device while operating underneath the critical capillary number.

\subsubsection{Limitations and future considerations}

Although capable of controllably merging droplets under a variety of flow conditions, the merging chamber is limited by its reliance on high-surface-tension systems. The design relies on the ability of the droplet to maintain its shape while experiencing high shear rates at the bypass junction and therefore is susceptible to the breaking of droplets and their subsequent buildup in the bypass junctions. The 
buildup of the dispersed phase may remain in the dead volume zone of the bypass channel especially when the bypass channel is large, which in turn reduces the amount of oil which can bypass the trapped droplet and therefore can hinder the functionality of the merging design. An example of this is visible in the bypass channels in Figs. 1 and 2. Although these conditions will affect the determination of $\mathrm{Ca}_{\text {crit }}$, there will still exist an operational regime where the merging is predictable, but the buildup of aqueous solution in the bypass channel would require mechanical perturbations to clear the bypass. For example, we used a pencil tip to press the bypass channel in where an aqueous droplet stayed, and then the droplet was pushed out of the bypass channel.

It has been demonstrated that the merging chamber design is capable of merging a varying number of droplets depending on the size of input droplets entering the chamber, although a few limitations currently exist. To address these limitations, the design can be modified in a particular manner to prevent the buildup of the dispersed phase within the bypass channel by providing a suitable means for the flow to passively clear out the bypass channel. Also, because the merging occurs at a point of stagnant flow, the system can easily be integrated with active methods such as electric fields to force the droplets to merge with lowsurface-tension systems. This design is therefore able to be integrated in a varicty of different microfluidics systems, both active and passive, for the facilitation of biochemical reactions within microfluidics LOC systems.

\section{Conclusions}

One of the most critical components of an assay is the control of reagent concentration. Therefore, ensuring that the droplets can effectively merge and initiate reactions is the most critical component for droplet-based LOC devices. The question of how to effectively merge droplets can have a multitude of solutions, each with their own advantages and limitations. A key advantage provided by the design proposed is its capability of balancing the requirements for reaction volume and the desired number of different reagents and their relative concentrations while maintaining the design simplicity. From the experimental results, it was demonstrated that the merging design was capable of merging between 6 and 2 droplets with $L_{\mathrm{J}} / L$ between 0.18 and 0.65 , respectively. It was also determined that $\mathrm{Ca}_{\text {crit }}$ for each design is proportional to the BRR. The relationship allows for the estimation of the working range of flow rates for the design and can be manipulated by the $W_{\text {by }}^{*}$ and $L^{*}$ parameters. The output droplet volume can be restricted and controlled through the manipulation of the $L^{*}$ parameter, and the consistency in product volumes allows for accurate representation of measured data.

Acknowledgements The authors gratefully acknowledge the support from Canada Natural Sciences and Engineering Council of Canada, Canada Foundation for Innovation, Canada Research Chair program and Advanced Electrophoresis Solutions Ltd, through grants to Dr. Carolyn L. Ren. A. Brukson acknowledges the support of Waterloo Institute of Nanotechnology through a fellowship.

\section{References}

Abate AR, Hung T, Mary P, Agresti JJ, Weitz. DA (2010) Highthroughput injection with microfluidics using picoinjectors. Proc Natl Acad Sci 107:19163-19166

Basu AS (2013) Droplet morphometry and velocimetry (DMV): a video processing software for time-resolved, label-free tracking of droplet parameters. Lab Chip 13:1892-1901

Boybay MS, Jiao A, Glawdel T, Ren CL (2013) Microwave sensing and heating of individual droplets in microfluidic devices. Lab Chip 13:3840-3846

Bremond N, Thiam A, Bibette J (2008) Decompressing emulsion droplets favors coalescence. Phys Rev Lett 100:024501

Christopher GF, Bergstein J, End NB, Poon M, Nguyen C, Anna SL (2009) Coalescence and splitting of confined droplets at microfluidic junctions. Lab Chip 9:1102-1109

Dewan A, Kim J, Mclean RH, Vanapalli SA, Karim MN (2012) Growth kinetics of microalgae in microfluidic static droplet arrays. Biotechnol Bioeng. 109:2987-2996

Frenz L, Harrak AEI, Pauly M, Colin SB, Griftiths AD, Baret JC (2008) Droplet-based microreactors for the synthesis of magnetic iron oxide nanoparticles. Angew Chem Int Ed 47:6817-6820

Garstecki P, Fuerstman MJ, Stone HA, Whitesides GM (2006) Formation of droplets and bubbles in a microfluidic T-junction-scaling and mechanism of break-up. I ab Chip 6:437-446

Glawdel T, Elbuken C, Ren C (2011) Passive droplet trafficking at microfluidic junctions under geometric and flow asymmetries. I.ab Chip 11:3774-3784

Glawdel T, Elbuken C, Ren C (2012) Droplet formation in microfluidic T-junction generators operating in the transitional regime. I. Experimental observations. Phys Rev E 85:016322

Gu H, Duits MHG, Mugele F (2011) Droplets formation and merging in two-phase flow microfluidics. Int J Mol Sci 12:2572-2597

Guo MT, Rotem A, Heyman JA, Weitz DA (2012) Droplet microfluidics for high-throughput biological assays. Lab Chip 12:2146-2155

He M, Edgar JS, Jeffries GD, Lorenz RM, Shelby JP, Chiu DT (2005) Selective encapsulation of single cells and subcellular organelles into picoliter- and femtoliter-volume droplets. Anal Chem 77:1539-1544

Hung LH, Choi KM, Tseng W, Tan Y, Shea KJ, Lee AP (2006) Alternating droplet generation and controlled dynamic droplet fusion in microfluidic device for CdS nanoparticle synthesis. I.ab Chip 6:174-178

Isgor PK, Marcali M, Keser M, Elbuken C (2015) Microfluidic droplet content detection using integrated capacitive sensors. Sensor Actuat B Chem 210:669-675

Jin BJ, Kim YW, Lee Y, Yoo JY (2010) Droplet merging in a straight microchannel using droplet size or viscosity difference. J Micromech Microeng, 20:035003

Kaler KVIS, Prakash R (2014) Droplet microfluidics for chip-based diagnostics. Sensors (Basel) 14:23283-23306 
Lee M, Collins JW, Aubrecht DM, Sperling RA, Solomon L, Ha JW, Yi GR, Weitz. DA, Manoharan VN (2014) Synchronized reinjection and coalescence of droplets in microfluidics. Lab Chip 14:509-513

Li ZG, Ando K, Yu JQ, Liu AQ, 7hang JB, Ohl CD (2011) Fast ondemand droplet fusion using transient cavitation bubbles. Lab Chip 11:1879-1885

Lin BC, Su YC (2008) On-demand liquid-in-liquid droplet metering and fusion utilizing pneumatically actuated membrane valves. J Micromech Microeng 18:115005

Link D, Anna S, Weitz D, Stone H (2004) Geometrically mediated breakup of drops in microfluidic devices. Phys Rev Lett 92:54503

Liu K, Ding H, Chen Y, Thao X (2007) Droplet-based synthetic method using microflow focusing and droplet fusion. Microfluid Nanolluid 3:239-243

I.iu Z, Ou J, Samy R, Glawdel T, Huang T, Ren CI. (2008) Side-byside comparison of disposable microchips with commercial capillary cartridges for application in capillary isoelectric focusing with whole column imaging detection. I ab Chip 8:1738-1741

Lorenz RM, Fiorini GS, Jeffries GD, Lim DS, He M, Chiu DT (2008) Simultaneous generation of multiple aqueous droplets in a microfluidic device. Anal Chim Acta 630:124-130

Luong T, Nguyen N, Sposito A (2012) Thermocoalescence of microdroplets in a microfluidic chamber. Appl Phys Lett 100:254105

Mazutis L, Baret JC, Griffiths AD (2009) A fast and efficient microfluidic system for highly selective one-to-one droplet fusion. Lab Chip 9:2665-2672

Miller OJ, El Harrak A, Mangeat T, Baret JC, Frenz L, El Debs B, Mayot E, Samuels ML, Rooney EK, Dieu P, Galvan M, Link DR, Grifliths AD (2012) High-resolution dose-response screening using droplet-based microfluidics. Proc Natl Acad Sci 109:378-383

Niu X, Gulati S, Edel JB, deMello NJ (2008) Pillar-induced droplet merging in microfluidic circuits. Lab Chip 8:1837-1841

Song H, Tice JD, Ismagilov RF (2003) A microfluidic system for controlling reaction networks in time. Angew Chemie 115:791-796
Tabeling P (2014) Recent progress in the physics of microfluidics and related biotechnological applications. Curr Opin Biotech 25:129-134

Tan YC, Fisher JS, Lee AI, Cristini V, Lee AP (2004) Design of microfluidic channel geometries for the control of droplet volume, chemical concentration, and sorting. Lab Chip 4:292-298

Tan YC, Ho YL, Lee AP (2007) Droplet coalescence by geometrically mediated flow in microfluidic channels. Microfluid Nanofluid 3:495-499

Trivedi V, Doshi A. Kurup GK, Ereifej E, Vandevord PJ, Basu AS (2010) A modular approach for the generation, storage, mixing. and detection of droplet libraries for high throughput screening. Lab Chip 10:2433-2442

van Steijn V, Kleijn CR, Kreutzer MT (2010) Predictive model for the size of bubbles and droplets created in microfluidic T-junctions. Lab Chip 10:2513-2518

Venkatachalam C, Weidenhof B, Krămer M, Maier WF, Herminghaus S, Seemann R (2010) Optimized droplet-based microfluidics scheme for sol-gel reactions. Lab Chip 10:1700-1705

Wheeler AR, Throndset WR, Whelan RJ, Leach AM, Zare RN, Liao YH, Farrell K, Manger ID, Daridon A (2003) Microfluidic device for single-cell analysis. Anal Chem 75:3581-3586

White IFM (1991) Viscous fluid flow, 2nd edn. McGraw-Ilill, New York

Xiong B, Ren K, Shu Y, Chen Y, Shen B, Wu H (2014) Recent developments in microlluidics for cell studies. Adv Mater 26:5525-5532

Xu L, Lee H, Panchapakesan R, Oh KW (2012) Fusion and sorting of two parallel trains of droplets using a railroad-like channel network and guiding tracks. I.ab Chip 12:3936-3942

Yang L, Wang K, Tan J, Lu Y, Luo G (2011) Experimental study of microbubble coalescence in a T-junction microfluidic device. Microfluid Nanofluid 12:715-722

Yoon DH, Jamshaid $\Lambda$, Ito J, Nakahara $\Lambda$, Tanaka D, Akitsu T, Sekiguchi T, Shoji S (2014) Active microdroplet merging by hydrodynamic flow control using a pneumatic actuator-assisted pillar structure. Lab Chip 14:3050-3055 